EPiC Series in Computing
Volume 64, 2019, Pages 31-40
Proceedings of 28th International Conference
on Software Engineering and Data Engineering

\title{
Motivational Game-Theme Based Instructional Module for Teaching Binary Tree and Linked List
}

\author{
Sarika Rajeev ${ }^{1}$ and Sharad Sharma ${ }^{1}$ \\ ${ }^{1}$ Department of Computer Science, Bowie State University, Bowie, MD, 20715, USA \\ rajeevs1027@students.bowiestate.edu, ssharma@bowiestate.edu
}

\begin{abstract}
Game theme based learning modules will revolutionize education because it increases motivation and engagement of students as they learn interactively. This study is aimed to assess the perceived motivation and engagement of undergraduate students for game theme based learning in introductory programming courses. This paper presents the design, implementation, and evaluation of a game theme-based instructional (GTI) module to teach linked list and binary tree data structure. We have used FDF (fourdimensional framework) with a minor extension for the design and development of GTI modules. The design of the GTI module is modeled on the constructive approach of learning. The purpose of this paper is to overcome the intellectual crisis by providing a new way of thinking and learning. We have evaluated the GTI modules based on the five components of Science Motivation Questionnaire II (SMQII). The results of the evaluation of GTI modules show that the students feel self-determined and motivated towards their learning and career.
\end{abstract}

\section{Introduction}

There is a need to change the traditional passive method of teaching to an active method of teaching such as game and simulation-based learning. Many students choose to use unethical means (cheating, plagiarism, collaboration on assignments) to get a good grade. So, we need to provide a better learning environment by increasing the student's motivation towards learning. The game theme-based instructional (GTI) modules prepare the learners to think critically, and the students can adopt new challenges of the relevant knowledge. Many researches indicate that students are more tempted towards unethical means in education because of few reasons, which includes: (1) excess workload, (2) trying to get a good grade at any cost (3) introduction to new concepts, and (4) time pressure placed upon students $[1,2]$. This paper presents a novel and exciting methodology of learning which includes ethics in future education by motivating the students towards learning. Game theme based learning helps to 
improve the problem-solving skills of students and produce better learning outcomes [3, 4]. We had designed and developed GTI modules to teach the linked list and binary tree data structure. Sharma [57], indicates that GTI modules help the students to have a better understanding of concepts by engaging them more towards learning. Woods [8], states that the simulation element of games has educational potential. Squire [9], mentioned that the games have educational potential regarding both subjective and social perspective.

We have used a proven four-dimensional framework [10] to assess the perceived learning outcomes and effectiveness of GTI modules. The first dimension of the framework emphasizes on "context" of learning. The educational benefits that, the students receive in one context may differ from another context. So, the context is an essential factor to consider during the design and development of GTI modules. The context for learning depends on both the micro level and macro level factors. The microlevel factors include (1) access to equipment, (2) technical support, and (3) availability of specific resources. The macro level factors for GTI modules include (1) historical, (2) political and, (3) economical. The context for GTI modules is classroom-based or lab-based. However, it is not limited to use in classrooms; the students can use the GTI modules for self-study.

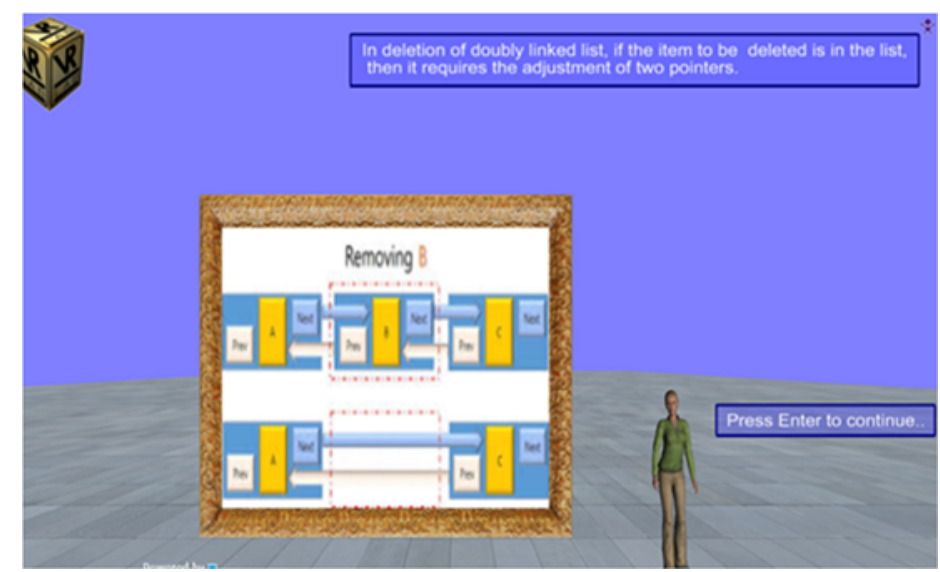

Figure 1: Virtual Instructor in GTI module for teaching doubly linked list

The second dimension of the framework focuses on the attributes of learning, such as the learner's background, age, learning style, and preferences. For the GTI modules, the learners are undergraduate and graduate students of the computer science department. The age of learners (users of GTI modules) should be between 18 to 26 years. Many studies indicate that the adolescent spends more time with software games than an adult [11]. We have developed GTI modules to motivate young learners towards learning and nurturing them into an intellectual adult. The third dimension of the framework emphasizes the approach of learning. The GTI modules are designed using the constructive approach of learning. The learning process is more fair and unbiased when the students construct their knowledge through experience with GTI modules. The fourth dimension of the framework emphasizes on the internal representation of the GTI modules. The GTI module is highly interactive and non-immersive. The students' hesitation (shyness) to ask their queries to a real instructor is a major cause of poor learning outcomes and student's unethical approach to learning. We have included a virtual instructor (figure 1) in the GTI modules which help the students during gameplay.

We have evaluated GTI modules using Science Motivation Questionnaire II (SMQII). SMQII is a questionnaire to find the motivation of students. It has five components which include: (1) Intrinsic motivation, (2) Self-determination, (3) Self-efficacy, (4) Career motivation and, (5) Grade motivation. The results of the user study indicate that the students get motivated towards learning and produce better learning outcomes. 


\section{Related Work}

\subsection{Motivation and enthusiasm in education}

Recent studies show that the idea which states that, "there is no relation between emotion and learning," becomes obsolete [12,13]. According to Damasio [12], emotions not only determine the intelligence but also provide a means to intelligence. According to Goleman [13], if the students are angry, uneasy or distressed, then they will not learn efficiently. So, for better learning outcomes it is necessary to evaluate the motivation and enthusiasm of the learners. Using a self-report approach, we can evaluate the motivation and enthusiasm of students for a specific teaching methodology. Del Soldato [14] introduced a motivational modeler and a motivational planner to measure the level of motivation when students used traditional intelligent tutoring system for learning new concepts.

De Vincente [15] states that motivation is an essential aspect of the educational software, and it can be analyzed by using a method named 'motivational diagnosis.' Matsubara and Nagamachi [16], state about instructional planning to increase motivation. The likeability is considered as a factor of motivation. According to Keller [17], it is not always possible that if the instruction (traditional lecture by the instructor) is of good quality, then it will motivate the students. There are many pieces of research have been done to deal with motivation [14-16]. Vincente and pain [15] presented an empirical study, which showed a significant amount of knowledge related to the motivational diagnosis.

In $\mathrm{AI}$ and educational research, the pupil's motivation is virtually unexplored. In the traditional method of teaching teachers or instructors get cues from the students through a different communication medium such as posture and facial expression. However, many of these cues are unrelated which makes it challenging to evaluate the motivation of students. Vincente and Pain [15] designed a study in which an instructor is asked to conclude pupils motivational state. For this study, they used prerecording of students' interaction with the system. The researchers concluded that the evaluation of motivation would be easier if they use the video recording of the students' interaction [15]. The duration of time the students spend with the software is also noteworthy to evaluate the motivation.

\subsection{A constructive approach to learning}

Constructivism refers to the belief that the learners construct knowledge for themselves via interaction. Constructivists focus more on an understanding of knowledge through experience and less on the verifying the concept [18]. The learners are the devoted creator of their knowledge based on the previous idea and new experience. Constructivists believe that the learners are not an empty container to be filled with loads of knowledge; instead, they can make the meaning of the knowledge. The consequence of the theory is twofold.

1. Learners in thinking mode for learning

2. Learners do not have knowledge which is not related to their experience.

The constructivist theory appears in response to behaviorism and cognitivism. Researchers believe that a human is not a robot so, he could not be programmed [19]. Constructivists assert that human brain plays an active role in learning. In other words, the learners are much more actively involved than teachers or peers to learn or understand a particular concept. Constructivism refers to both learning theory and epistemology [18]. Regarding learning theory, the constructivists suggest that learners construct meaning. In epistemology, constructivists suggest that knowledge is constructed through discussion or interaction with peers.

In constructivism, students construct and reconstruct the concept with an attempt to match with the experience. The new concept is matched with the student's prior knowledge, pre-conception, and misconception. If the new material is consistent, then it is considered to be learned, and if it is contradictory, then it is considered as "it cannot be learned." 


\section{Experimental Design of GTI Modules}

This section discusses a brief overview of the activity system and four-dimensional framework, which we have used to design the GTI modules.

\subsection{Activity system for GTI modules}

According to Kuutti [20], activity is a form of doing which leads to an object. Various activities are distinguished from each other based on their objects. We have incorporated a primary individual activity system, in our proposed GTI modules. We have utilized the Kuutti's activity system [20] in GTI modules as follows:

1. Tool: GTI modules have non-immersive 3D-interface. It has a high level of interactivity. In GTI modules users can interact with the virtual instructor to clarify the concepts.

2. Subject: Undergraduate students from computer science department who have taken introductory programming courses.

3. Object: The objective for GTI modules is to clarify the concept (binary tree and linked list). We want to motivate and engage the students in learning linked list and binary tree concepts.

4. Rules: We have utilized a constructive approach to learning, in GTI modules. The students construct their knowledge using a gaming metaphor. We have used Bloom's taxonomy in the design of GTI modules.

5. Community: Our proposed GTI modules do not support group study.

6. Division of labor: We have designed GTI modules to be used in the classroom or computer lab. However, students can use GTI modules at their home during leisure time.

\subsection{Framework for the evaluation of GTI module}

We have used a proven framework for finding the effectiveness of GTI modules. The first dimension of the framework focuses on the context where the learning takes place. We want to use GTI modules as a supplement in classroom-based learning. The context for GTI modules is undergraduate classes of computer science. We need limited technical support to use the GTI modules. We also considered the macro level factors of the context, such as (1) Historical, (2) Political and, (3) Economical. The instructors/tutors must have a specific background such as (1) minimal technical knowledge of computer usage (2) familiar with virtual reality games. We have developed the GTI modules for classroom-based context, but the use of GTI modules is not limited to the classroom. This educational software tool (GTI module) is platform independent. So, this software (GTI module) can be used outside the classroom. In other words, the GTI modules are outdoor accessible and; are not limited to classroom or computer lab

The second dimension of the framework, which we have used to identify the effectiveness of GTI module, focuses upon the attributes of learners. From the phrase "learner's specification," the first question arises in our mind is: who is the learner? Before designing or developing the GTI modules (educational software), we need to clarify the target audience or users of the GTI modules. The users or learners of GTI modules are undergraduate students from the computer science department. However, the GTI modules can be used in graduate classes of introductory programming. GTI modules do not support group study. The GTI modules are aimed to support individual learners only. According to Provenzo [21], children and adolescent are more fascinated by games. We developed GTI modules for an adolescent of ages 18 years to 26 years. The background and the learning history of the students is also an important factor which affects the effectiveness of GTI modules. The GTI modules support the students of different learning background/style. There is the various level of games in each GTI modules (binary trees or linked list). The students can choose the game according to their preference 
and learning style. There is a range of differential learners. The game theme-based instructional module is basically for college students, but this tool can be used by others who want to learn programming concepts (binary trees and linked list) in an informal setting.

The third dimension of the framework focuses on pedagogic consideration. We need to decide the pedagogic models and approaches to learning before we design the GTI modules. The game themebased instructional modules are developed with constructive theory in mind. The constructive learning theory states that learners construct knowledge by experiencing it in the real world. When the undergraduate students of computer science department use GTI modules for learning the concepts of binary trees and linked list; they interact with the game theme-based instructional module and learn through their experience with the gaming metaphor. The learning activities of GTI modules emphasis on the clarification of the concepts of binary trees/linked list and to produce better learning outcomes. The learning activities include: (1) Interacting with the virtual instructor, (2) Navigating through the GTI modules and, (3) Playing the games of GTI modules. We have used briefing/non-briefing to reinforce learning outcomes.

The fourth dimension of the framework emphasizes the mode of representation or tool for use. Undeniably, this dimension focuses on the processes of learning. This dimension considers model, approaches, and theories to support learning. GTI modules use a medium level of fidelity based upon the use of non-immersive 3D interfaces. The game theme-based instructional modules have a high level of interactivity. GTI modules have buttons through which the learners interact with the virtual environment. Students or learners use mouse clicks and keyboard commands for interacting with the GTI modules. The way the students can interact with the GTI modules are listed as follows:

1. Students can ask for help using the buttons. They can go to the main menu and switch to another game anytime.

2. During gameplay, if the students need to learn about the dedicated concept, then the students can end game ask the virtual instructor (using mouse and keyboard command) for the better understanding of the concepts they want to learn.

3. Students can skip the tutorial if they are already familiar with the concept and directly move to the game of their choice.

4. Students can also answer the popped-up questions during gameplay. These questions are rewarding, and there is a scoreboard which displays the student's score. So, the students can evaluate themselves while learning.

The learning activities and outcomes are achieved partly by playing games and partly by interacting with the virtual instructor. The GTI modules use a medium level of realism regarding the 3D nonimmersive virtual environment and 3D model of virtual instructor. The GTI modules can be easily converted into an immersive virtual environment having a high level of realism using virtual reality hardware devices such as Oculus Rift.

\section{GTI Implementation}

The GTI modules were implemented in two phases, such as: (1) We created the 3D-models using 3d-max and sketch-up. (2) we exported the 3D models in Vizard and added game mechanics using Python.

\subsection{Implementation of the linked list and binary tree module}

In GTI modules, the user is greeted with a brief overview of the concepts related to the linked list/binary trees. A 3D avatar of the virtual tutor explains the basic concepts related to a linked list/binary tree in brief. Users are guided on the screen to proceed to the next steps. The students can press "enter key" to go to the next screen. There is a real-life example of the train to show the linked list. Students 
can add cars to the trains and construct their knowledge 'how to create a linked list. We also implemented a real-world example of the circular linked list by creating a group of a woman standing in a circle. As shown in figure 2, all woman held each other hand; students can visualize there is no null pointer (defined end) and can construct their knowledge about the difference in a linked list and circular linked list. We have used assorted interface elements to make the linked list/binary tree modules more attractive.

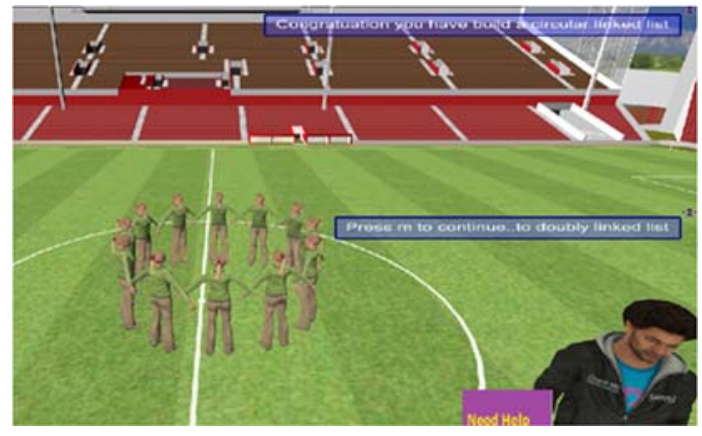

Figure 2: A real-life example of a circular linked list

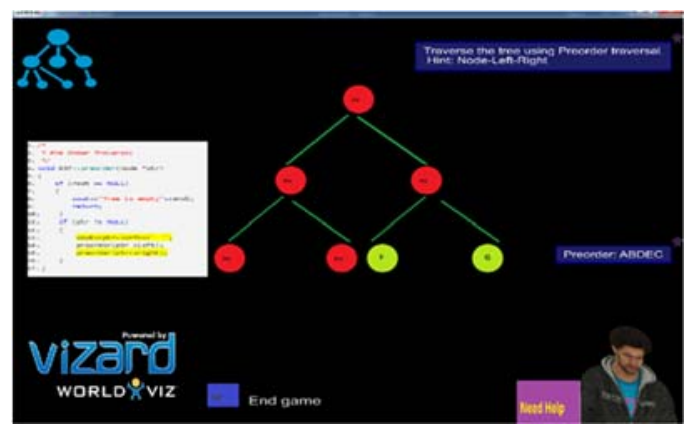

Figure 3: In-order traversal of binary tree

The games of GTI modules are rewarding so; the students get some idea of his/her learning progress. The award system of GTI modules can motivate the students towards learning. There is a steady virtual instructor in the GTI modules who support the learners to navigate through the GTI modules and provide help in the understanding of definition and example of the binary tree and linked list. The virtual instructor aimed to support the learning of various concepts of binary trees and linked list. The students can ask for help anytime from the virtual instructor. We have included a high-level view of gameplay by adding a pseudo code on the screen. Students can visualize the programming concept while they use the GTI modules (figure 3).

\subsection{GUI for a linked list and binary tree modules}

The GUI for gameplay in the GTI module to teach linked list is given in figure 4. We have utilized the following GUI to make our modules more appealing:

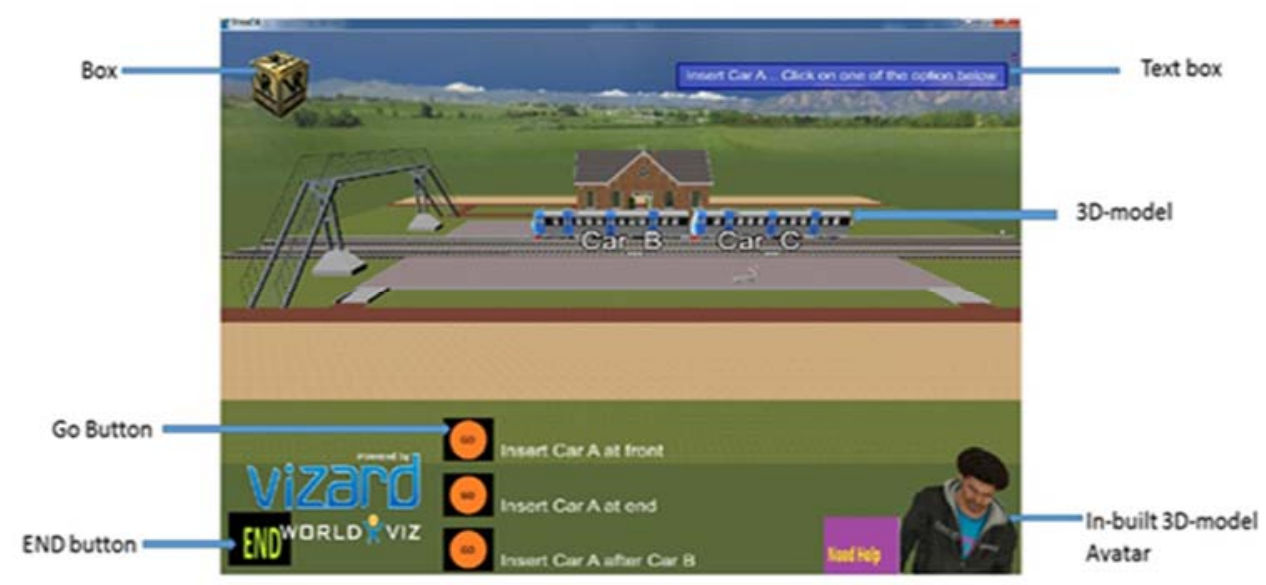

Figure 4: Assorted interface elements of GTI modules to teach linked list 
- Buttons: We have used a range of buttons to make the gameplay more revealing, such that (1) GO button, (2) Help button, (3) Next button and (4) End button. We have used "GO button" for choosing between given options. The "help" button is used for clarifying the queries of the users. We have added the "help" button to make the GTI modules more usable. We have exploited the "Next" button to go on the next screen and; the "End" button to end the gameplay and move on main menu page/screen.

- Boxes: We have incorporated boxes (VR logo) to shows the linked list/binary tree module is virtual reality-based gameplay.

- $\quad$ 3D- models: We have exploited 3D-models to make the linked list and binary tree modules more appealing. We have created the 3D models using 3D max and SketchUp and imported these models in Vizard. We have also used in-built avatars of vizard to make the gameplay more likable.

- Text: We have incorporated various "text boxes" to make the GTI modules more informative. The "text boxes" make the GTI modules more usable as the user can take help from the "text." The users also receive a compliment using the text boxes such as: "good job" and "congratulation."

- Scoreboard: There is a scoreboard incorporated in the gameplay to display the score of the users. When the users complete an activity or challenge, then the score is incremented by "50" points, and the scoreboard displays the score of the candidate.

- Images of pseudo code: The pseudocode of the linked list is displayed on the screen. The goal is to provide the learners with a high-level view of the fundamentals of the linked list. The GTI modules should motivate and engage the students in learning linked list concepts.

\section{Simulation and Results}

Our primary goal of this research is to provide a better learning environment by increasing the motivation of the students toward learning. 57 college students (42 undergraduate and 15 graduate students) helped us in evaluating the GTI modules in the spring semester. We demonstrated the technique to use the GTI modules to the students before the evaluation process. Then the students played with the module and completed a post-survey. The survey questions were based on SMQII questionnaire. The survey contained five questions each for each of the five components of SMQII.

For the statistical analysis of motivation towards learning (the concepts of the binary tree and linked list) with the GTI modules, we have integrated the T-test. We have collected the data using pre- and post-survey from introductory programming classes. We compare the data collected from pre-and postsurvey using the t-test. We have used a five-point Likert scale ranging from "strongly agree" to "strongly disagree". Table 1 shows the mean value of motivation of students (for both the pre- and post-survey) in the learning of binary tree and linked list concepts. We have analyzed the collected data (table 2) using. T-test with the hypothesis:

H1: There is a significant difference between the motivation of students when they use the GTI module to learn the binary tree and linked list.

We have tested our hypothesis is true using the null hypothesis $\mathrm{H} 0$.

H0: There is no significant difference between the motivation of students in pre-and post-survey.

\begin{tabular}{|l|l|l|}
\hline Motivation & Pre-survey & post-survey \\
\hline The Linked list and binary tree concept I learn is relevant to my life. & 2.65 & 3.40 \\
\hline Learning Linked list and binary tree concept is interesting. & 2.61 & 3.59 \\
\hline I am curious about applications of the Linked list and binary tree concept. & 2.39 & 3.55 \\
\hline I enjoy learning Linked list and binary tree concept. & 2.58 & 3.51 \\
\hline I am confident I will do well on the Linked list \& binary tree concept tests. & 2.52 & 3.63 \\
\hline $\begin{array}{l}\text { I am confident I will do well on labs and projects based on the Linked list } \\
\text { and binary tree concept. }\end{array}$ & 2.42 & 3.56 \\
\hline
\end{tabular}




\begin{tabular}{|l|l|c|}
\hline $\begin{array}{l}\text { I believe I can master knowledge and skills based on the Linked list and } \\
\text { binary tree concept. }\end{array}$ & 2.19 & 3.69 \\
\hline I am sure I can understand Linked list and binary tree concept. & 2.10 & 3.63 \\
\hline I use strategies to learn Linked list and binary tree concept well. & 2.45 & 3.46 \\
\hline I spend a lot of time learning Linked list and binary tree concept. & 2.58 & 3.41 \\
\hline I prepare well for Linked list and binary tree concept tests and labs. & 2.55 & 3.41 \\
\hline I study hard to learn the Linked list and binary tree concept. & 2.35 & 3.44 \\
\hline Getting a good grade is important to me. & 1.43 & 4.28 \\
\hline I think about the grade I will get. & 1.57 & 4.22 \\
\hline Scoring high on tests and labs matters to me. & 1.37 & 4.31 \\
\hline Knowing the concepts in depth will give me a career advantage. & 1.73 & 3.85 \\
\hline I will use problem-solving skills in my career. & 1.33 & 3.85 \\
\hline
\end{tabular}

Table 1: Mean value of motivation to learn in pre-and post-survey

The result of the above t-test for motivation to learn binary tree and the linked list is summarized in Table 2. A total number of items which we need to compare using T-test was 17. Table 2 illustrates the mean values of the motivation to learn the binary tree and linked list for each of the groups (pre-and post-survey). The mean value of motivation to learn in post-survey was 3.69 whereas the mean value of motivation to learn in pre-survey was 2.16 .

\begin{tabular}{|l|l|l|}
\hline & post-survey & pre-survey \\
\hline Mean & 3.692804 & 2.165908 \\
\hline Variance & 0.094652 & 0.230832 \\
\hline Observations & 17 & 17 \\
\hline Hypothesized Mean Difference & 0 & \\
\hline Df & 27 & \\
\hline $\mathrm{t} \mathrm{Stat}$ & 11.03492 & \\
\hline $\mathrm{P}(\mathrm{T}<=\mathrm{t})$ one-tail & $8.24 \mathrm{E}-12$ & \\
\hline $\mathrm{t}$ Critical one-tail & 1.703288 & \\
\hline $\mathrm{P}(\mathrm{T}<=\mathrm{t})$ two-tail & $1.65 \mathrm{E}-11$ & \\
\hline $\mathrm{t}$ Critical two-tail & 2.051831 & \\
\hline
\end{tabular}

Table 2: t-Test: Two-Sample Assuming Unequal Variances

Additionally, it includes the respective results of the variances of both pre- and post-survey based on motivation to learn linked list and binary tree. The result involves the degree of freedom, the T values and the critical value of the t-tests. Results show that the degree of freedom (df) for the t-test is 27 , tvalue ( $\mathrm{t} \mathrm{Stat}$ ) is 11.0349 and the critical value (t Critical two-tail) is 2.051831 . The results of the $\mathrm{t}$-test for the motivation have shown that $t$-value (11.0349) is greater than the critical value (2.051831). Additionally, the p-value (1.65E-11) of the t-test is lesser than the hypothesized p-value (0.05). So, we can reject the null hypothesis and; we accept the alternate hypothesis such that there is a significant difference in motivation to learn when we use GTI module for learning binary trees and linked list.

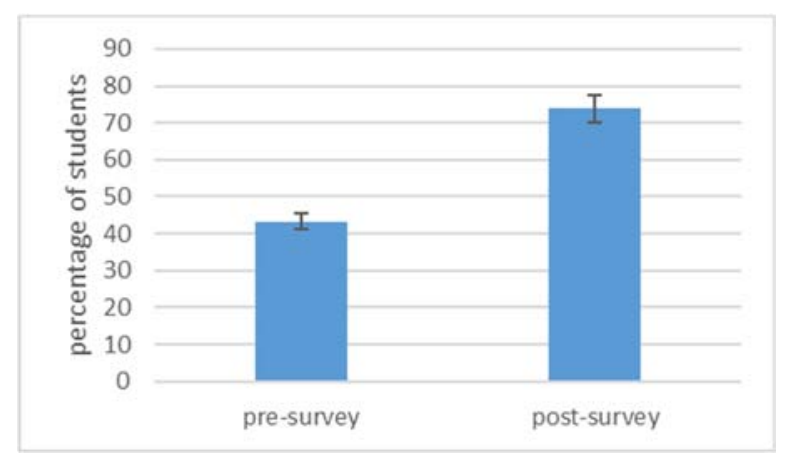

Figure 5: Mean value of motivation for pre- and post-survey 


\section{Conclusions}

We have designed and implemented GTI modules to motivate the students towards learning. We have used the four-dimensional framework with minor extension for the design of GTI modules. The GTI modules can be included in the regular classroom as a supplement of teaching. We have evaluated GTI modules using SMQII questionnaire. The GTI modules were evaluated in the spring semester with 57 students (15 graduate and 42 undergraduate). The outcomes of the statistical analysis (t-test) for the motivation have shown that the p-value (1.65E-11) of the t-test is lesser than the hypothesized p-value $(0.05)$. So, we can conclude that students felt motivated and engaged in learning. The result of the empirical evaluation shows that $74 \%$ of students (refer Figure 5) got motivated when they used GTI modules for learning.

\section{Acknowledgments}

This work is funded in part by the ARL Award: W911NF1820224.

\section{References}

[1] L. Dömeová1 and A. Jindrova, "Unethical Behavior of the Students of the Czech University of Life Sciences," International Education Studies; Vol. 6, No. 11; ISSN 1913-9020 E-ISSN 1913-9039 Published by Canadian Center of Science and Education, 2013.

[2] Z. Rustam, A. Naseem, I. Waqar, A. Mehmood, Yasir. "Unethical practices in university system: pattern and causes," Investigation from Pakistan (KPK). Science Series Data Report. 4. 23., 2012.

[3] J. Stigall, S. Sharma, "Virtual Reality Instructional Modules for Introductory Programming Courses," Proceedings of IEEE Integrated STEM Education Conference (ISEC), pages: 33- 41, DOI:978-1-5090-5379-7/17, Princeton, New Jersey, Saturday, March 11, 2017.

[4] S. Sharma, E. Ossuetta, "Virtual Reality Instructional Modules in Education Based on Gaming Metaphor," IS\&T International Symposium on Electronic Imaging (EI 2017), in The Engineering Reality of Virtual Reality Proceedings Papers, Hyatt Regency San Francisco Airport, Burlingame, California, 29 January- 2 February 2017.

[5] S. Sharma, J. Stigall, S. Rajeev, "Game-Theme Based Instructional Module for Teaching ObjectOriented Programming," Proceedings of the IEEE International Conference on Computational Science and Computational Intelligence (CSCI), Las Vegas, USA, Page 252-257, DOI 10.1109/CSCI.2015.3, December 7-9, 2015.

[6] S. Rajeev, S. Sharma, A. Sahu, "Game Theme Based Instructional Module to teach Binary Trees Data Structure," Proceedings of ISCA 26th International Conference on Software Engineering and Data Engineering, pp. 13-18, San Diego, CA, USA, October 2-4, 2017.

[7] J. Stigall, S. Sharma, "Usability and Learning Effectiveness of Game-Themed Instructional (GTI) Module for Teaching Stacks and Queues," Proceedings of IEEE SoutheastCon 2018, Hilton Bay Front, St. Petersburg, FL, USA, April 19 - 22, 2018

[8] S. Woods, Loading the dice: The challenge of serious video games. Game Studies, 4(1)http://www.gamestudies. org/0401/woods/, Last accessed 15th March 2005.

[9] K. Squire, "Cultural framing of computer/video games." Game Studies, 2(1), See http://www.gamestudies.org/ 0102/squire/, Last accessed 15th March 2005.

[10] S. Freitas, M. Oliver, "How can exploratory learning with games and simulations within the curriculum be most effectively evaluated?” Computers \& Education 46, 249-264, 2006. 
[11] A. Bruckman, \& A. Brandlow, HCI for kids. In J. Jacko \& A. Sears (Eds.), Human-Computer Interaction Handbook, (pp. 428-440, Hillsdale, NJ: Lawrence Erlbaum. 2003.

[12]A. Damasio, Descartes' Error: Emotion, Reason, and the Human, Brain, New York: Grosset/Putnam. 1994.

[13]D. Goleman, Emotional Intelligence, New York: Bantam Books. 1995.

[14] T. Soldato, Detecting and Reacting to the learner's motivational state. In C. Frasson, G. Gauthier \& G. I. McCalla (Eds.), Intelligent Tutoring Systems: Proceedings of ITS'92 - Montréal, pp. 567574, New York: Springer-Verlag 1992.

[15]A. De Vincente, \& H. Pain, Informing the detection of the students' motivational state: An empirical study. In S. A. Cerri, G. Gouarderes, \& F. Paraguacu (Eds.), Intelligent Tutoring Systems 2002, LNCS, 2363 (pp. 932-943). Berlin, Heidelberg: Springer-Verlag, 2002.

[16] Y. Matsubara \& Y. Nagamachi, Motivation system and human model for intelligent tutoring. In C. Frasson, G. Gauthier, \& A. Lesgold (Eds.), Proceedings of the 3rd International Conference on ITSs (pp. 139-147). Berlin: Springer-Verlag, 1996.

[17] J. M. Keller, Motivational design of instruction. In C. M. Reigeluth (Ed.), Instructional-Design Theories and Models: An Overview of their Current Status, pp. 386-434, Hillsdale, NJ: Lawrence Erlbaum Associates, 1983.

[18] S. Papert, "Mindstorms: Children, Computers and Powerful Ideas" Basic Books, New York, 1980.

[19] L. Harasim, Learning Theory and Online Technologies, 2012.

[20] K. Kuutti, Activity theory as a potential framework for human-computer interaction research. In B. A. Nardi (Ed.), Context and consciousness: Activity theory and human-computer interaction, pp. 17-44, Cambridge, MA: The MIT Press, 1996. 